\title{
Drift of Scroll Waves in Thin Layers Caused by Thickness Features: Asymptotic Theory and Numerical Simulations
}

\author{
I. V. Biktasheva, ${ }^{1}$ H. Dierckx ${ }^{2}$ and V. N. Biktashev ${ }^{3}$ \\ ${ }^{1}$ Department of Computer Science, University of Liverpool, Liverpool L69 3BX, United Kingdom \\ ${ }^{2}$ Department of Mathematical Physics and Astronomy, Ghent University, 9000 Ghent, Belgium \\ ${ }^{3}$ College of Engineering, Mathematics and Physical Sciences, University of Exeter, Exeter EX4 4QF, United Kingdom
}

(Received 11 August 2014; published 11 February 2015)

\begin{abstract}
A scroll wave in a very thin layer of excitable medium is similar to a spiral wave, but its behavior is affected by the layer geometry. We identify the effect of sharp variations of the layer thickness, which is separate from filament tension and curvature-induced drifts described earlier. We outline a two-step asymptotic theory describing this effect, including asymptotics in the layer thickness and calculation of the drift of so-perturbed spiral waves using response functions. As specific examples, we consider drift of scrolls along thickness steps, ridges, ditches, and disk-shaped thickness variations. Asymptotic predictions agree with numerical simulations.
\end{abstract}

DOI: 10.1103/PhysRevLett.114.068302

PACS numbers: 82.40.Ck, 02.70.-c, 05.10.-a, 82.40.Bj

Spiral waves in two dimensions (2D) and scroll waves in three dimensions (3D) are regimes of self-organization observed in physical, chemical, and biological spatially extended dissipative systems with excitable or selfoscillatory properties [1]. A particularly important example is the reentrant waves of excitation underlying arrhythmias in the heart [2]. In nature, 2D systems often are very thin 3D layers of the medium, so the dynamic fields vary only slightly in the transmural direction. The geometry of a layer affects the dynamics of scroll waves via the well-known phenomena of scroll wave filament tension [3] and surface curvature of the layer [4], which cause scroll waves to drift to or from thinner regions and more curved regions, respectively. There are, however, effects not reducible to these phenomena and rather related to sharp features of the layer thickness. Figure 1 shows a paradoxical example of a scroll wave with a positive filament tension first attracted towards the thicker part of the layer and then drifting along the thickness step. There is experimental evidence that sharp thickness variations can play a significant role in atrial fibrillation $[5,6]$.

In this Letter, we present an asymptotic theory of drift of scroll waves caused by variations of layer thickness. Predictions of this theory are quantitatively confirmed by direct numerical simulations for two selected archetypical models, one excitable and one self-oscillatory. We demonstrate that sharp variations can produce drifts that are not reducible to filament tension and surface curvature. The details of these drifts depend on the reaction-diffusion kinetics, as well as the size, geometry, and position of the thickness feature. A typical motif, observed for both selected models, is that a scroll is first attracted towards a sharp thickness variation and then drifts along or around it.

We start from a generic homogeneous isotropic reactiondiffusion system in $3 \mathrm{D}$,

$$
\mathbf{v}_{t}=\mathbf{f}(\mathbf{v})+\mathbf{D} \nabla^{2} \mathbf{v}
$$

where $\mathbf{v}=[u(\vec{r}, t), v(\vec{r}, t)]^{T}, \vec{r}=(x, y, z)$. In numerical examples, we use the excitable FitzHugh-Nagumo (FHN) system [8], with kinetics

$$
\mathbf{f}:\left[\begin{array}{l}
u \\
v
\end{array}\right] \mapsto\left[\begin{array}{c}
\alpha^{-1}\left(u-u^{3} / 3-v\right) \\
\alpha(u+\beta-\gamma v)
\end{array}\right]
$$

for $\alpha=0.3, \beta=0.68, \gamma=0.5$, and $\mathbf{D}=\operatorname{diag}(1,0)$, and the self-oscillatory Oregonator model of the BelousovZhabotinsky reaction [9], with kinetics

$$
\mathbf{f}:\left[\begin{array}{l}
u \\
v
\end{array}\right] \mapsto\left[\begin{array}{c}
p^{-1}\left(u(1-u)-f v \frac{u-q}{u+q}\right) \\
u-v
\end{array}\right]
$$

for $p=0.1, f=1.5, q=0.002$, and $\mathbf{D}=\operatorname{diag}(1,0.6)$ (see the Supplemental Material [7]).

We consider the system of Eq. (1) in a thin layer, $z \in\left[z_{\min }(x, y), z_{\max }(x, y)\right], \quad(x, y) \in \mathbb{R}^{2}$, with no-flux boundaries at $z=z_{\min }$ and $z=z_{\max }$. Let $H(x, y) \equiv$ $z_{\max }(x, y)-z_{\min }(x, y)$ and $0<H(x, y) \leq \mu \ll 1$. Then,

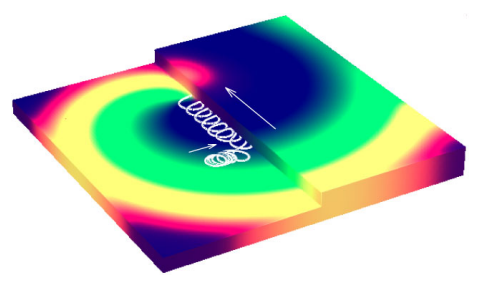

FIG. 1 (color online). Surface view of a scroll wave in a thin layer of excitable medium described by the FitzHugh-Nagumo system [Eqs. (1), (2)], with a stepwise variation of thickness. The white curve is the trace of the vortex filament appearing at the top surface (Supplemental Material [7]). 
$\mathbf{v}(x, y, z, t)=\mathbf{u}(x, y, t)+\mathcal{O}\left(\mu^{2}\right)$ (Supplemental Material [7]), and Eq. (1) in the leading order in $\mu$ reduces to the following $2 \mathrm{D}$ approximation:

$$
\mathbf{u}_{t}=\mathbf{f}(\mathbf{u})+\mathbf{D} \frac{1}{H(x, y)} \nabla \cdot[H(x, y) \nabla \mathbf{u}]+\mathcal{O}\left(\mu^{2}\right) .
$$

We rewrite Eq. (4) in the form

$$
\mathbf{u}_{t}=\mathbf{f}(\mathbf{u})+\mathbf{D} \nabla^{2} \mathbf{u}+\epsilon \mathbf{h}(\mathbf{u}, x, y, \nabla)
$$

where

$$
\epsilon \mathbf{h}=\epsilon \mathbf{D}(\nabla K) \cdot(\nabla \mathbf{u}), \quad \epsilon K=\ln H .
$$

Equations (5) and (6) will be treated as a perturbation problem with the formal small parameter $\epsilon$, distinct from the small parameter $\mu$. So, for Eq. (5) we assume the existence of a rigidly rotating spiral wave solution $\mathbf{U}$ at $\epsilon=0$.

In what follows, we explicitly calculate scroll wave drift for three given geometries corresponding to abrupt changes in domain thickness, i.e., a thickness step, ditch, and circular bulge. First, we consider a step in thickness, as in Fig. 1,

$$
H(x, y)= \begin{cases}H_{+}, & x>x_{s}, \\ H_{-}, & x<x_{s} .\end{cases}
$$

Since $K=K(x)$, one has $\epsilon \mathbf{h}=\epsilon \mathbf{D} K_{x} \mathbf{u}_{x}$. With $\Theta$, the Heaviside step function, we have $\epsilon K=\ln \left(H_{-}\right)+$ $\epsilon \Theta\left(x-x_{s}\right), \epsilon=\ln \left(H_{+} / H_{-}\right)$, such that

$$
\epsilon \mathbf{h}=\epsilon \delta\left(x-x_{s}\right) \mathbf{D} \mathbf{u}_{x} .
$$

Equations (12), (13), and (14) of Ref. [10] predict the drift velocity $\epsilon F(\vec{R})=\epsilon\left(F_{x}+\mathrm{i} F_{y}\right)$ as overlap integrals of translational response functions

$$
\begin{aligned}
\frac{d \vec{R}}{d t} & =\epsilon \vec{F}(\vec{R})=\epsilon\left(F_{x}, F_{y}\right), \\
F(\vec{R}) & =\int_{0}^{\infty} \oint \mathbf{W}(r, \theta)^{\dagger} \boldsymbol{\alpha}(r, \theta ; \vec{R}) d \theta r d r \\
\boldsymbol{\alpha}(r, \theta ; \vec{R}) & =\oint e^{-i \phi} \tilde{\mathbf{h}}(\mathbf{U}, r, \theta, \phi) \frac{d \phi}{2 \pi}
\end{aligned}
$$

where $\tilde{\mathbf{h}}$ is the perturbation $\mathbf{h}$, calculated for $\mathbf{u}=\mathbf{U}$ and considered in the frame corotating with the spiral, $\mathbf{W}$ are (translational) response functions of the spirals, and $\dagger$ stands for conjugate transposed. Counterclockwise rotating spirals and their response functions calculated for the two selected models using DXSPIRAL [11] (Supplemental Material [7]) are illustrated in Fig. 5 in Ref. [7]; change of chirality of the spirals corresponds to complex conjugation of $\mathbf{W}, \boldsymbol{\alpha}$, and $F$. Evaluation of the integral of Eq. (11) with an account of Eq. (8) and the coordinate transformations $\mathbf{h}(\vec{R}, t)=\tilde{\mathbf{h}}(r, \theta, \phi), \quad \vec{R}=(X, Y), \quad \vec{r}=(x, y)$, $d=X-x_{s}, \quad \theta=\vartheta(\vec{r}-\vec{R})+\phi, \quad r=\rho(\vec{r}-\vec{R}), \quad x+i y \equiv$ $\rho(\vec{r}) \exp [i \vartheta(\vec{r})]$ gives

$$
\boldsymbol{\alpha}= \begin{cases}0, & r \leq|d|, \\ \frac{\mathbf{D e}^{-i \theta}}{\pi \sqrt{r^{2}-d^{2}}}\left[\frac{d^{2}}{r^{2}} \mathbf{U}_{r}-\frac{i\left(r^{2}-d^{2}\right)}{r^{3}} \mathbf{U}_{\theta}\right], & r>|d| .\end{cases}
$$

Equations (10) and (12) define the specific force produced by the thickness step, which depends only on the distance between the current spiral center and the step and is an even function about the position of the step,

$$
\begin{gathered}
F(\vec{R})=S(d), \quad d \equiv X-x_{s}, \\
S(-d)=S(d)=S_{x}(d)+\mathrm{i} S_{y}(d) .
\end{gathered}
$$

The components of the function $S(d)$ for the two selected models are shown in Figs. 2(b) and 2(e). An important feature is the zeros of $S_{x}$ for $d= \pm d^{*}$ in both models. Assuming without loss of generality that $x_{s}=0$, the drift of a spiral wave is then described asymptotically by

$$
\frac{d X}{d t}=\epsilon S_{x}(X), \quad \frac{d Y}{d t}=\epsilon S_{y}(X), \quad \epsilon=\ln \left(\frac{H_{+}}{H_{-}}\right) .
$$

Figure 2 illustrates predictions of the theory for the case of a thickness step and their comparison with the direct numerical simulations of both the 2D thickness-reduced system (4) and the full 3D system (1). Numerical simulations for both selected models were done with BEATBOX [12] (Supplemental Material [7]). The relevant attractor for Eq. (15) is

$$
X=-d^{*}, \quad Y=Y_{0}+\epsilon S_{y}\left(-d^{*}\right) t,
$$

where $S_{x}\left(-d^{*}\right)=0, S_{x}{ }^{\prime}\left(-d^{*}\right)<0$. That is, in both models the spirals attach to the step at its thinner side and drift along with the speed $\left|\epsilon S_{y}\left(-d^{*}\right)\right|$. The speed of the drift is proportional to $\epsilon=\ln \left(H_{+} / H_{-}\right)$, and the direction of the drift depends on the spiral chirality: compare Figs. 2(a) and 2(d).

As a second geometry, let us consider the following thickness profile: for some $x_{\ell}<x_{r}$,

$$
H(x, y)= \begin{cases}H_{o}, & x<x_{\ell}, \\ H_{i}, & x_{\ell}<x<x_{r}, \\ H_{o}, & x_{r}<x,\end{cases}
$$

which means a "ridge" for $H_{i}>H_{o}$ and a "ditch" for $H_{i}<H_{o}$. This case is easily reduced to the previous because $H(x, y)=H_{i}+\left(H_{o}-H_{i}\right)\left[\Theta\left(x-x_{\ell}\right)-\Theta\left(x-x_{r}\right)\right]$; 


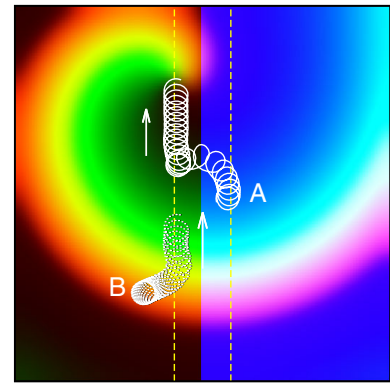

(a)

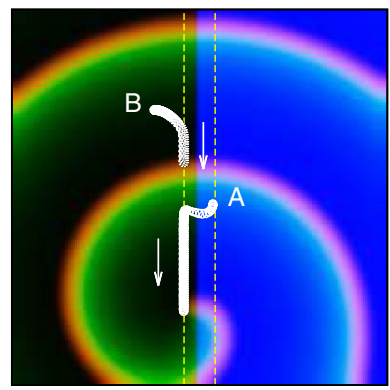

(d)

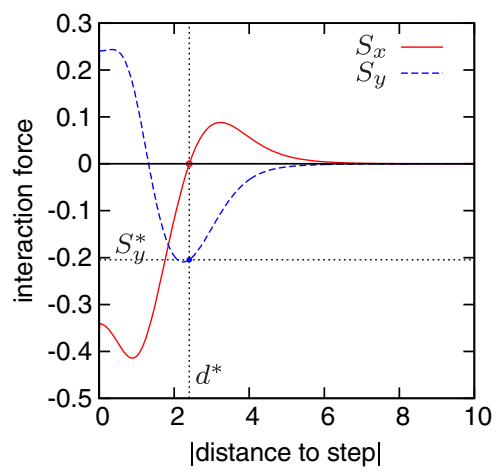

(b)

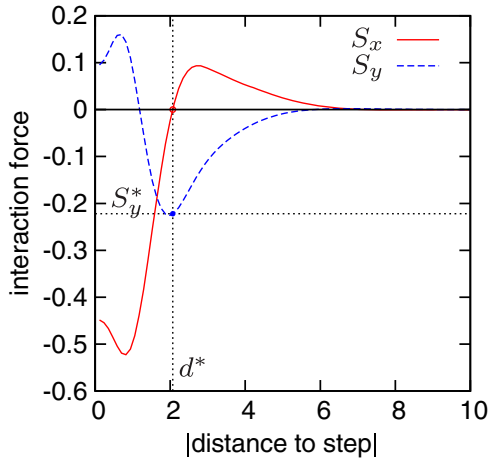

(e)

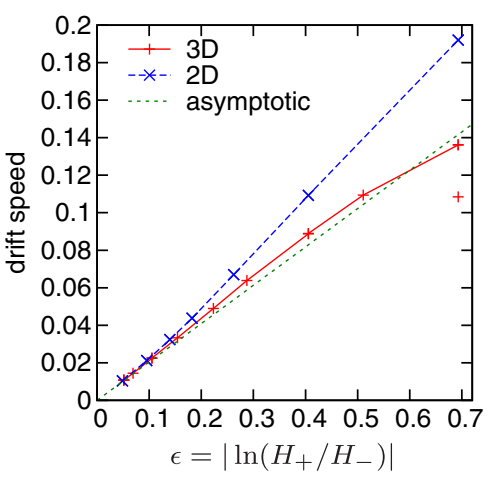

(c)

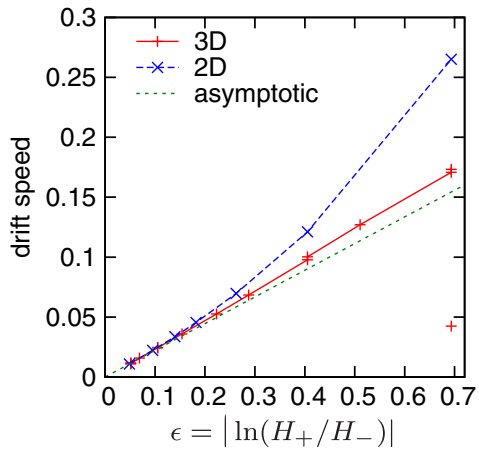

(f)

FIG. 2 (color online). Asymptotic theory vs numerical simulations for interaction of a scroll wave with a thickness step, for the FitzHugh-Nagumo system [panels (a)-(c)] and the Oregonator model [panels (d)-(f)]. [(a), (d)] Spiral wave snapshot (red color component: $u$ field, green color component: $v$ field, blue color component: $H$ field), with the previous tip path (solid white line) starting at A, another path starting at a different point (B), and loci $d= \pm d^{*}$ (dashed yellow lines), in the 2D system (4). [(b), (e)] Components of the specific force $S$ (14) calculated for counterclockwise spirals. [(c), (f)] Drift speed along the step in the 3D system (1), the 2D system (4), and the asymptotic predicted by Eq. (16).

hence, the formal perturbation is $\epsilon \mathbf{h}=\epsilon\left[\delta\left(x-x_{\ell}\right)-\right.$ $\left.\delta\left(x-x_{r}\right)\right] \mathbf{D u} \mathbf{u}_{x}$, where $\epsilon=\ln \left(H_{i} / H_{o}\right)$. Let $x_{\ell}=x_{s}-w / 2$, $x_{r}=x_{s}+w / 2$. We use the linearity of Eqs. (9)-(11) and the previous result to get the interaction force in the form

$$
\begin{gathered}
F(\vec{R})=T(d ; w)=-T(-d ; w), \quad d \equiv X-x_{s}, \\
T=T_{x}+i T_{y}=S\left(d+\frac{w}{2}\right)-S\left(d-\frac{w}{2}\right) .
\end{gathered}
$$

Figures 3(a) and 3(b) show the components of $T(d ; w)$ for two selected values of the ridge width $w$, illustrating a pitchfork bifurcation of $T_{x}$ roots. The bifurcation condition $T_{x}(d ; w)=\partial_{d} T_{x}(d ; w)=0$, observation that the bifurcation happens at $d^{*}=0$ and evenness of $S(d)$ gives the critical value of the width implicitly as the condition $S_{x}{ }^{\prime}\left(w^{*} / 2\right)=0$. For the FHN system, there are two positive roots for $S_{x}{ }^{\prime}(\cdot)$ [see Fig. 2(b)], the smaller giving $w^{*} \approx 1.769$. For a ditch $(\epsilon<0)$, this predicts neutrally stable equilibria along the middle line of the ditch if $w>w^{*}$ [Fig. 3(b)] and a drift along either side of the ditch, in one direction or the other, depending on the initial condition, if $w<w^{*}$ [Fig. 3(a)].

Figures 3(c) and 3(d) illustrate the drift along a cuneiform ditch, i.e., a ditch with almost constant but slowly varying width. The coordinate scale along the ditch is the same in both panels, with the bifurcation width $w^{*}$ designated by the dashed horizontal line. We see that below this line the spiral wave drifts in accordance with the theory and slows down markedly in the vicinity of this line. It does not stop completely but proceeds further, albeit at a much slower speed, which may be seen as a transient pinning. This slow drift is due to the "wedging" effect of the varying width: at $w \geq w^{*}$, the forces from the two opposite steps, constituting the banks of the ditch, do not compensate each other exactly due to the angle between them. To estimate roughly the associated correction, let the wedge angle be $\psi \ll 1$. Then the wedge-forced component of the drift speed at the bifurcation point is $2 \epsilon S_{x}\left(w^{*}\right) \sin (\psi / 2) \approx \epsilon S_{x}\left(w^{*}\right) \psi$. For the simulation shown in Figs. 3(c) and 3(d), we have $\psi \approx 0.03$, and $S_{x}\left(w^{*}\right) \approx$ 0.4142 ; hence, the drift speed $\epsilon \psi S_{x}\left(w^{*}\right) \approx 0.002266$. This wedge-forced drift speed is represented by the dotted line in Fig. 3(d) and agrees well with the simulations. If the initial 


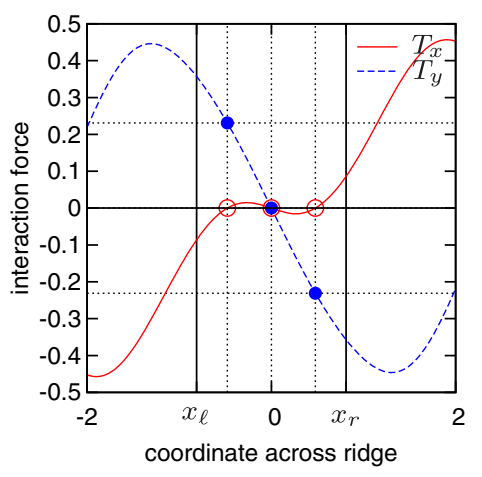

(a)

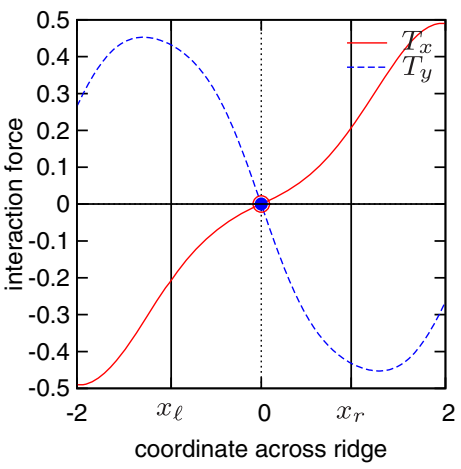

(b)

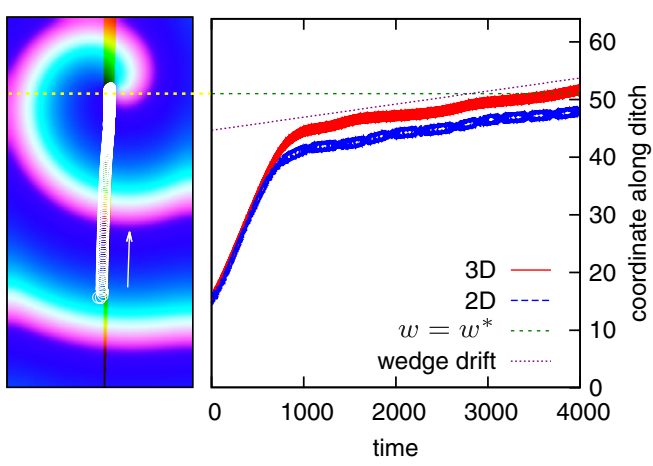

(c)

(d)

FIG. 3 (color online). Results of asymptotic theory of interaction with a ridge or ditch and comparison with simulations, for the FitzHugh-Nagumo system (2). [(a), (b)] Specific interaction force $T(d)$ (19) calculated for counterclockwise spirals: (a) for $w=1.621<w^{*}$; (b) for $w=1.953>w^{*}$. (c) Spiral wave snapshot (red color component: $u$ field, green color component: $v$ field, blue color component: $H$ field), with the previous tip path (white line), drifting along a cuneiform ditch, of width 0.24 at the lower end, linearly growing to 2.16 at the upper end, box size $32 \times 64$, and $H_{o} / H_{i}=1.2$. (d) Coordinate of the spiral tip along the ditch as a function of time. The horizontal dashed line shows the location of the ditch width $w=w^{*}$ corresponding to the point of the pitchfork bifurcation of $T(d ; w)$. The slope of the dotted line represents the slow drift speed due to the sides of the ditch being nonparallel.

position of the spiral is where $w \gtrsim w^{*}$, then it undergoes only the slow, wedge-forced drift from the start (not shown).

Drift caused by ridge or ditch features may help in understanding the dynamics of scroll waves in atrial geometry, for instance, around pectinate muscles [6]. Another feature, specifically analyzed in Ref. [6] by numerical simulations, was a circular bulge. To see what our theory can say about that, let us consider, thirdly, a thickness perturbation of the form

$$
H(x, y)=H_{0}\left(1+\epsilon \Theta\left(R_{d}^{2}-\left(x-x_{d}\right)^{2}-\left(y-y_{d}\right)^{2}\right)\right),
$$

i.e., thickening (for $\epsilon>0$ ) or thinning (for $\epsilon<0$ ) in a disk-shaped area of radius $R_{d}$. Then we have

$$
\alpha=\frac{e^{i \vartheta_{0}} e^{-i \theta} \mathbf{D}}{\pi r \ell \sqrt{1-\kappa^{2}}}\left[\left(\ell \kappa^{2}+r \kappa\right) \mathbf{U}_{r}-\frac{i \ell\left(1-\kappa^{2}\right)}{r} \mathbf{U}_{\theta}\right]
$$

for $\vec{r} \in\left(\left|R_{d}-\ell\right|, R_{d}+\ell\right)$, and $\boldsymbol{\alpha}=0$ otherwise. Here, $\ell e^{i \vartheta_{0}}=\left(x_{d}-X\right)+i\left(y_{d}-Y\right)$ represents the vector from the current spiral center $(X, Y)$ to the bulge center $\left(x_{d}, y_{d}\right)$, and $\kappa=\left(R_{d}^{2}-\ell^{2}-r^{2}\right) /(2 r \ell)$. Hence, the interaction force is

$$
F_{x}+\mathrm{i} F_{y}=e^{i \vartheta_{0}}\left[F_{r}(\ell)+i F_{a}(\ell)\right] .
$$

The radial $F_{r}(\ell)$ and the azimuthal $F_{a}(\ell)$ components calculated for the Oregonator model (3) for an arbitrarily chosen disk radius $R_{d}$ are shown in Fig. 4(a). We observe there is a root of $F_{r}(\ell)$ at $\ell=\ell^{*} \approx 4.023$ and the corresponding value of the specific force $F_{a}^{*}=F_{a}\left(\ell^{*}\right) \approx$ 0.1055 predicts long-term behavior of a spiral starting from an appropriate initial condition as "meander" or "orbital movement" along a circle of radius $\ell^{*}$ with the linear speed $\epsilon F_{a}^{*}$ and an orbit period of $2 \pi \ell^{*} /\left(\epsilon F_{a}^{*}\right) \approx 1314$. Figure 4(b) compares these predictions with results of $2 \mathrm{D}$ and $3 \mathrm{D}$ numerical simulations at $\epsilon=\log (1.2)$. This result is similar to the case considered phenomenologically in Ref. [6] and is analogous to the "orbital motion" described in Ref. [13] for localized parametric heterogeneities.

To summarize, the movement of transmural scroll waves through thin layers of excitable media of varying thickness can be approximately described by thickness-averaged two-dimensional equations, and a corresponding 2D perturbation theory can be successfully applied within its limits. Our theory shows the propensity of scrolls to interact with sharp features of the layer geometry. In the examples

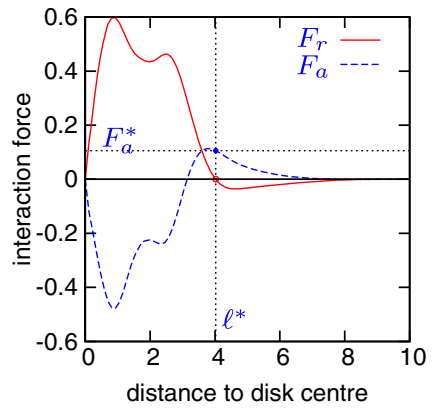

(a)

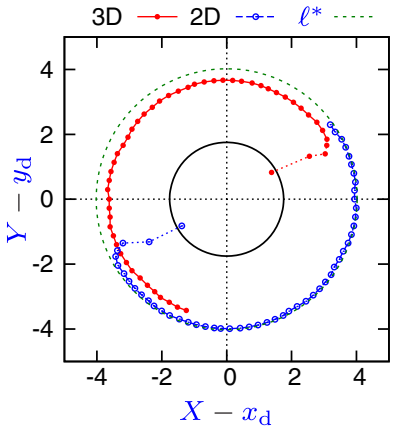

(b)
FIG. 4 (color online). Interaction of a spiral with a disk-shape bulge in Oregonator model. (a) Components of the interaction force calculated according to Eqs. (10), (11), (20), (21), for $R_{d}=225 / 1280 \approx 1.756$. (b) Tip trajectories in simulations of duration corresponding to half of predicted orbiting period (lines as indicated by the legend), together with initial transients (thin dotted lines). Green dashed circle: the theoretically predicted stationary orbit of the spiral center drift. Black solid circle: the boundary of the bulge. 
considered, this interaction caused a scroll to position itself at a certain distance from a sharp feature and drift along or around it with the speed determined by the feature's magnitude, measured by the relative variation of the thickness. This is distinct from and not reducible to previously known geometric effects such as filament tension or curvature-induced drift and is completely independent from other factors that may cause drift, such as parametric inhomogeneities or external forcing (see e.g., Ref. [10]). Interaction with sharp features can manifest nontrivial attractor structures, depending on the geometric parameters. These predictions should be immediately testable in experiments with the Belousov-Zhabotinsky reaction, can be used in experiments, for instance, in precision positioning of scrolls, and may have important implications for understanding the evolution of reentrant waves of excitation in the heart, particularly in atria, which have an abundance of geometric features. For instance, our results give a theoretical explanation and, hence, suggest a universal character of scroll wave "anchoring" and "meandering" caused by thickness variations, which are implicated in the perpetuation of atrial fibrillation [6].

H. D. is supported by FWO-Flanders (Belgium). The development of DXSPIRAL and BEATBOX was supported by EPSRC Grants No. EP/D074789/1 and No. EP/I029664/1 (UK).

[1] A. M. Zhabotinsky and A. N. Zaikin, in Oscillatory Processes in Biological and Chemical Systems, edited by E. E. Selkov, A. A. Zhabotinsky, and S. E. Shnol (Nauka, Pushchino, 1971), p. 279; M. A. Allessie, F. I. M. Bonke, and F. J. G. Schopman, Circ. Res. 33, 54 (1973); F. Alcantara and M. Monk, J. Gen. Microbiol. 85, 321 (1974); N. A. Gorelova and J. Bures, J. Neurobiol. 14, 353 (1983); B. F. Madore and W. L. Freedman, Am. Sci. 75,
252 (1987); S. Jakubith, H. H. Rotermund, W. Engel, A. von Oertzen, and G. Ertl, Phys. Rev. Lett. 65, 3013 (1990); J. Lechleiter, S. Girard, E. Peralta, and D. Clapham, Science 252, 123 (1991); T. Frisch, S. Rica, P. Coullet, and J. M. Gilli, Phys. Rev. Lett. 72, 1471 (1994); M. C. Cross and P. C. Hohenberg, Rev. Mod. Phys. 65, 851 (1993).

[2] F. H. Fenton, E. M. Cherry, and L. Glass, Scholarpedia 3, 1665 (2008).

[3] V. N. Biktashev, A. V. Holden, and H. Zhang, Phil. Trans. R. Soc. A 347, 611 (1994).

[4] H. Dierckx, E. Brisard, H. Verschelde, and A. V. Panfilov, Phys. Rev. E 88, 012908 (2013).

[5] T. J. Wu, M. Yashima, F. Xie, C. A. Athill, Y. H. Kim, M. C. Fishbein, Z. Qu, A. Garfinkel, J. N. Weiss, H. S. Karagueuzian, and P.S. Chen, Circ. Res. 83, 448 (1998).

[6] M. Yamazaki, S. Mironov, C. Taravant, J. Brec, L. M. Vaquero, K. Bandaru, U. M. R. Avula, H. Honjo, I. Kodama, O. Berenfeld, and J. Kalifa, Cardiovasc. Res. 94, 48 (2012).

[7] See the Supplemental Material at http://link.aps.org/ supplemental/10.1103/PhysRevLett.114.068302 for details of asymptotic and numerical procedures.

[8] R. FitzHugh, Biophys. J. 1, 445 (1961); J. Nagumo, S. Arimoto, and S. Yoshizawa, Proc. IRE 50, 2061 (1962); A. T. Winfree, Chaos 1, 303 (1991).

[9] R. J. Field and R. M. Noyes, J. Chem. Phys. 60, 1877 (1974); J. J. Tyson and P. C. Fife, ibid. 73, 2224 (1980).

[10] I. V. Biktasheva, D. Barkley, V. N. Biktashev, and A. J. Foulkes, Phys. Rev. E 81, 066202 (2010).

[11] I. V. Biktasheva, D. Barkley, V. N. Biktashev, G. V. Bordyugov, and A. J. Foulkes, Phys. Rev. E 79, 056702 (2009), http://www.csc.liv.ac.uk/ ivb/software/DXSpiral .html.

[12] R. McFarlane and I. V. Biktasheva, in BCS International Academic Conference "Visions of Computer Science" (Imperial College London, 2008), http://empslocal.ex.ac .uk/people/staff/vnb262/software/BeatBox/.

[13] V. N. Biktashev, D. Barkley, and I. V. Biktasheva, Phys. Rev. Lett. 104, 058302 (2010). 